\title{
ALGEBRAS SPLIT BY A GIVEN PURELY INSEPARABLE FIELD
}

\section{KLAUS HOECHSMANN}

1. Let $K$ be a field of characteristic $p \neq 0$. By a $p$-algebra we mean a central simple algebra over $K$ whose dimension is a power of $p$. Although it is known that such an algebra always has a purely inseparable (over $K$ ) splitting field $E$, the role played by $E$ in the structure of the algebra has not been clear. In this paper, we intend to show that essentially all $p$-algebras split by $E$ are obtained by a natural composition of two constituents: a certain purely inseparable field $\hat{E}$ containing $E$ and any abelian normal extension $N$ of $K$ whose Galois group is related, in a manner to be described, to the structure of $\hat{E}$. We must dwell a little on the nature of these ingredients.

Consider a subgroup $X$ of the multiplicative group $E^{*}$ of $E$ such that $X$ contains the multiplicative group $K^{*}$ of $K$. Such a group will be called regular, if any system of representatives of $X$ modulo $K^{*}$ is linearly independent over $K$. $E$ itself is called regular if it is additively generated by some regular subgroup of $E^{*}$, which in this case will be called a maximal regular subgroup. Just below the corollary for Theorem 2 in [2], it was shown that every finite purely inseparable extension $E$ can be further extended to a regular one $\hat{E}$ with the same exponent over $K$ and also finite. In what follows, we require $E$ to be regular. The field originally given may have to be enlarged to fulfill this condition, just as a separable field is extended to a normal one in the theory of crossed products. We assume, therefore that $\hat{E}=E$.

It follows at once from Theorem 1 of [2] that the group $G(X)$ $=X / K^{*}$ associated with a maximal regular subgroup $X$ of $E^{*}$ is independent of $X$. There is thus a unique $p$-group $G$ attached to $E$. The group $X$ is an extension of $K^{*}$ by $G$. Hence with the selection of a maximal regular group $X$ we obtain a cohomology class $\tilde{X} \in H^{2}\left(G, K^{*}\right)$. For the sequel let $X$ be fixed.

As for $N$, it will be a normal extension of $F$ with Galois group $\Gamma \simeq G$. However, $N$ need not be a field; in general it may be a direct sum of fields

$$
N=N_{1} \oplus \cdots \oplus N_{n},
$$

with a " $T$-group" $\Gamma$ of automorphisms, as defined in [1], i.e., a group of automorphisms satisfying the following three conditions.

Received by the editors July 6, 1962 . 
I. If $\sigma \in \Gamma$ and $\sigma$ keeps the elements of $N_{i}$ fixed (for any $i$ ), then $\sigma=1$.

II. $\Gamma$ is transitive on the set of fields $N_{1}, \cdots, N_{n}$.

III. If $a \in N$ and $a$ is fixed under all elements of $\Gamma$, then $a \in K$.

Teichmüller [3] proved that for $\alpha \in H^{2}\left(\Gamma, N^{*}\right)$ the crossed product $(N, \Gamma, \alpha)$ defined in the usual way is central simple over $K$ and has all the usual properties. The pair $(N, \Gamma)$ will be called a normal ring. ${ }^{1}$

We can now state our main theorem.

THEOREM 1. Let $A$ be a simple algebra of dimension $(E: K)^{2}$ over its center $K$. Then $E$ splits $A$ if and only if $A \simeq(N, \Gamma, \tilde{X})$ for some normal ring $(N, \Gamma)$ with $\Gamma \simeq G$.

Here $X$ is interpreted as an element of $H^{2}\left(\Gamma, K^{*}\right)$ as it can be because of the isomorphism between $G$ and $\Gamma$.

Before attempting to prove Theorem 1, we shall reformulate it to bring it into better accord with the crude version given at the outset. Given a normal ring $(N, \Gamma)$, consider an injection $\phi: \Gamma \rightarrow E^{*} / K^{*}$ such that $U_{\sigma \in \Gamma} \phi(\sigma)$ is a regular subgroup of $E^{*}$ (it is automatically maxi$\mathrm{mal})$. The regularity of $E$ guarantees the existence of such injections, since $\Gamma \simeq G$.

On the vector space $E \otimes_{K} N$, a multiplication is defined by demanding that

$$
(x \otimes u)(y \otimes v)=\left(x y \otimes u^{\sigma} v\right) \quad \text { if } y \in \phi(\sigma) .
$$

The resulting algebra will be denoted by $E \otimes_{\phi} N$.

THEOREM $1^{\prime}$. The class of algebras of the form $E \otimes_{\phi} N$ (for fixed regular E) coincides with that of p-algebras containing $E$ as a maximal commutative subring.

It is easily verified that the definition of $E \otimes_{\phi} N$ makes it isomorphic to $(N, \Gamma, \tilde{X})$ for suitable $X$. Indeed, suppose $\phi: \sigma \rightarrow x_{\sigma} K^{*}$. The nature of $\phi$ is such that the set $\left\{x_{\sigma} \mid \sigma \in \Gamma\right\}$ is a basis of $E$ over $K$, and hence a basis of $E \otimes_{\phi} N$ over $N$. If we write $x$ and $u$ instead of $(x \otimes 1)$ and $(1 \otimes u)$, respectively, the elements of $E \otimes_{\phi} N$ are of the form

$$
\sum_{\sigma \in \Gamma} x_{\sigma} u_{\sigma}
$$

where the $u_{\sigma}$ are arbitrary coefficients from $N$. The commuting rule (1) appears as:

$$
x_{\sigma} u=u^{\sigma} x_{\sigma} .
$$

'Often called "Galois algebra" and extensively studied in [5]. 
Finally, let $X=\bigcup_{\sigma \in \Gamma} x_{\sigma} K^{*} . X$ is a maximal regular subgroup of $E^{*}$, which can be thought of as an extension of $K^{*}$ by either $G$ or $\Gamma$. Taking the latter point of view, we may regard the factor set

$$
\alpha(\sigma, \tau)=\frac{x_{\sigma} x_{\tau}}{x_{\sigma \tau}}, \quad(\sigma, \tau \in \Gamma),
$$

as a representative of the cohomology class $\tilde{X}$.

We have exhibited the structure of a crossed product $(N, \Gamma, \tilde{X})$ in $E \otimes_{\phi} N$. For reasons of dimension and the simplicity of crossed products,

$$
E \underset{\phi}{\otimes} N \simeq(N, \Gamma, \tilde{X})
$$

Since $E$ is obviously contained in $E \otimes_{\phi} N$ (as the subring $E \otimes K$ ), we have also proved the "if" part of Theorem 1 .

2. For proving the second and more important part of Theorem 1, the theory of differential extensions, as worked out in [1], is needed. We recall briefly what it is about. Let $Z$ be a finite extension field of $K$ such that $Z^{p} \subseteq K, d$ be a derivation of $Z$ into $Z$, whose kernel is precisely $K$, and $f(x)$ be the minimal polynomial of $d$ over $K$. Given a central simple $Z$-algebra $A$, we can extend $d$ to a derivation $\bar{d}$ of $A$ into $A$ and find an element $c$ in the kernel of $\bar{d}$ such that $c a-a c$ $=f(\bar{d})(a)$ for all $a \in A$. The $K$-algebra $(A, \bar{d}, c)$, generated by $A$ and a symbol $u$ such that

$$
u a-a u=\bar{d} a, \quad \text { for all } a \in A,
$$

and

$$
f(u)=c
$$

is called a differential extension of $A$ by $\bar{d}$. It turns out that $(A, \bar{d}, c)$ is central simple over $K$ and contains $A$ as the centralizer of $Z$ and that, conversely, every such $K$-algebra is of the form $(A, \bar{d}, c+\gamma)$ with $\gamma \in K$. It is emphasized that $d$ and $\bar{d}$ can be chosen in various ways; in particular, $d$ can always be chosen to be regular, i.e., such that its proper vectors form a maximal regular subgroup of $Z^{*}$. (Note that $Z$, being of exponent $p$, is automatically regular.)

Now let $A$ be a crossed product of the normal ring $(M, \Delta)$ over $Z: M=M_{1} \oplus \cdots \oplus M_{m}$, each $M_{i}$ being a separable extension field of $Z$ and $\Delta$ being a $T$-group of automorphisms relative to $Z$. Let $\alpha \in Z^{2}\left(\Delta, M^{*}\right)$ be a 2 -cocycle defining $A$. We now impose a rather severe condition, namely, that the values $\alpha(\sigma, \tau)$ lie in some maximal 
regular subgroup of $Z^{*}$. It is well known that a derivation $d$ of $Z$ into $Z$ can be constructed whose group of proper vectors coincides with any given maximal subgroup of $Z^{*}$, in particular the one containing the values $\alpha(\sigma, \tau)$ (see, for example, [1, Proposition 1.3]). This derivation $d$ will be extended to $A$ as follows.

Since all elements of $M$ are separable over $Z, d$ has a unique extension to $M$. If $\left\{y_{\sigma} \mid \sigma \in \Delta\right\}$ is the usual $M$-basis for the crossed product $A$, the extensions $\bar{d}$ such that $\bar{d}(M) \subseteq M$ are defined by $d y_{\sigma}=y_{\sigma} \delta(\sigma)$, with $\delta: \Delta \rightarrow M$ satisfying

$$
d(\alpha(\sigma, \tau)) / \alpha(\sigma, \tau)=\delta(\sigma)^{\tau}-\delta(\sigma \tau)+\delta(\tau) .^{2}
$$

The function $\beta:(\sigma, \tau) \rightarrow d(\alpha(\sigma, \tau)) / \alpha(\sigma, \tau)$, as the "logarithmic derivative" of the multiplicative cocycle $\alpha$, is an additive cocycle, and (4) can be satisfied by setting

$$
\delta(\sigma)=\sum_{\rho \in \Delta} \beta(\rho, \sigma) a^{\rho \sigma}
$$

with any $a \in M$ for which $\sum_{\rho \in \Delta} a^{\rho}=1$. If $M$ were a field, the existence of such an element $a$ would be well known; in our case it can be found as follows. Let $\Delta_{1}$ be the subgroup of $\Delta$ leaving $M$ invariant. Clearly, $\Delta=\sigma_{1} \Delta_{1} \cup \sigma_{2} \Delta_{1} \cup \ldots \cup \sigma_{m} \Delta_{1}$, where $M_{1}^{\sigma_{i}}=M_{i}$. Furthermore, $\Delta_{1}$ induces on each subfield $M_{i}$ its Galois group over $Z$. We take an $a_{1}$ from $M_{1}$ whose trace is 1 . Then $a_{1}^{\sigma_{i}}$ has trace 1 in $M_{i}$; more precisely, if the elements of $M$ are represented in the form $\left(x_{1}, \cdots, x_{m}\right)$ with $x_{i} \in M_{i}$, we have

$$
\sum_{\rho \in \Delta}\left(a_{1}, 0, \cdots 0\right)^{\sigma_{i} \rho}=(0, \cdots, 1,0 \cdots)
$$

with the 1 in the $i$ th place. Hence

$$
\sum_{\rho \in \Delta}\left(a_{1}, 0, \cdots 0\right)^{\rho}=\sum_{i=1}^{m}(0, \cdots, 1,0 \cdots)=1 .
$$

The preceding paragraph is entirely independent of the condition imposed on $\alpha$, whose only purpose is to insure that certain things are separable over $K$. For, if $a_{1}$ is not $K$-separable, $a_{1}^{p}$ will surely be, and

$$
\sum_{\rho \in \Delta}\left(a_{1}^{p}\right)^{\rho}=\left(\sum_{\rho \in \Delta} a_{1}^{\rho}\right)^{p}=1 .
$$

In any case, $a_{1}$ can be chosen separable over $K$. Since each $\alpha(\sigma, \tau)$

2 This observation was made by G. Hochschild (Trans. Amer. Math. Soc. 80 (1955), 146). 
is a proper vector of the regular derivation $d$, each of the quotients $\beta(\sigma, \tau)$ is an element of $K$. Therefore, the function $\delta$ defined by (5) maps $\Delta$ into the maximal $K$-separable subring $M^{\prime}$ of $M$. Now we can state

THEOREM 2. In the notation introduced above, let $B=(A, \bar{d}, c)$ be any differential extension of the crossed product $A$. Then $B$ is again $a$ crossed product. More precisely, for suitable choice of $\bar{d}$, the ring $N$ generated in $B$ by $M^{\prime}$ and an element $u$ satisfying (2), is a direct sum of fields; the inner automorphisms induced in $B$ by certain proper vectors of $\bar{d}$ ( $\bar{d}$ being regarded as a linear transformation of $A$ over $\left.M^{\prime}\right)$ define a $T$-group of automorphisms on $N . N$ is a maximal commutative subring of $B$.

Proof. Let $\bar{d}$ be defined as an extension of the regular derivation $d$ of $Z$, exactly as above. $B$ is generated by $A$ and the element $u$ mentioned in the theorem, the latter satisfying the polynomial equation (3). Our first aim is to modify our choice of $\bar{d}$ so as to make $c$ lie in $M^{\prime}$.

Note that $c \in M$, because $c a-a c=f(\bar{d})(a)=0$, for $a \in M$, and because $M$ is maximal commutative in $A$. If $c$ is not separable over $K$, replace $\bar{d}$ by $\bar{d}^{p}$ and $u$ by $u^{p}$. It is easily checked that this change leaves all our conventions concerning $d$ intact. Now $f\left(u^{p}\right)=c^{p}$, which is $K$-separable.

Assume $c=c_{1}+\cdots+c_{m}, c_{i} \in M_{i}^{\prime}$. Then

$$
N=M^{\prime}[u] \simeq M^{\prime}[x] / f(x)-c \simeq \bigoplus_{i=1}^{m} M_{i}^{\prime}[x] / f(x)-c_{i} .
$$

Let $f(x)-c_{i}=\phi_{i 1}(x) \cdots \phi_{i r}(x)$ be a factorization into irreducible polynomials (it will become apparent that the number $r$ is the same for each $i$ ). Setting

$$
N_{i j}=M_{i}^{\prime}[x] / \phi_{i j}(x),
$$

we have, since $\phi_{i 1}(x), \cdots, \phi_{i r}(x)$ are all distinct because of the separability of $f(x)$,

$$
N \simeq \bigoplus_{i=1}^{m} N\left({ }_{i 1} \oplus \cdots \oplus N_{i r}\right)
$$

as claimed.

For an element $a \in A$, let $I a$ denote the map induced on $N$ by the inner automorphism $b \rightarrow a^{-1} b a$ of $B$.

Let $\left\{y_{\sigma} \mid \sigma \in \Delta\right\}$ be an $M$-basis of $A$ such that $x y_{\sigma}=y_{\sigma} x^{\sigma}$ for all $x \in M$. Finally denote by $W$ the group of proper vectors of $d$ in $Z^{*}$. 
We shall prove that the maps $I\left(y_{\sigma} z\right)$ with $z \in W$ form a $T$-group of automorphisms of $N$. In fact

$$
u\left(y_{\sigma} z\right)-\left(y_{\sigma} z\right) u=\bar{d}\left(y_{\sigma} z\right)=y_{\sigma} z(\delta(\sigma)+\lambda),
$$

where $\lambda$ is the proper value belonging to $z$, so that

$$
I\left(y_{\sigma} z\right): u \rightarrow u+\delta(\sigma)+\lambda .
$$

For $x \in M, I\left(y_{\sigma} z\right): x \rightarrow x^{\sigma} . I\left(y_{\sigma} z\right)$ is therefore an automorphism of $N$.

Before verifying conditions I, II, and III for a $T$-group, we make an observation:

$M_{i}=Z \otimes_{K} M_{i}^{\prime}$, and the restriction of $\bar{d}$ to $M_{i}$ is a regular derivation of $M_{i}$ over $M_{i}^{\prime}$ with the same proper values and vectors as $d$. Therefore we can use Theorem 6.1 of [1] to conclude that the maps $\{I z \mid z \in W\}$ form a $T$-group of automorphisms on $M_{i}^{\prime}[u]$.

(II) To map $N_{11}$ onto $N_{i j}$ we use $I\left(y_{\sigma} z\right)$ where $\sigma: M_{1} \rightarrow M_{i}$, and hence $M_{1}^{\prime} \rightarrow M_{i}^{\prime}$. Then $I y_{\sigma}$ maps $M_{1}^{\prime}[u]$ onto $M_{i}^{\prime}[u]$, and hence $N_{11}$ onto some $N_{i k}$. Since $I(W)=\{I z \mid z \in W\}$ is transitive on the fields $N_{i 1}, \cdots, N_{i r}$ in $M_{i}^{\prime}[u]$, the desired $z \in W$ can be found.

(I) Suppose $\tau=I\left(y_{\sigma} z\right)$ leaves $N_{11}$ elementwise fixed. There exist $z_{i} \in W(i=1 \cdots r)$ such that $\zeta_{i}=I z_{i}$ maps $N_{11}$ onto $N_{1 i}$. Since $W$ is in the center of $A$ and $y_{\sigma} \in A$, each $\zeta_{i}$ commutes with $\tau$. Let $x \in M_{1}^{\prime}[u]$, $x=x_{1}+\cdots+x_{r}$ with $x_{i} \in N_{1 i}$.

$$
x^{r}=\sum_{i=1}^{r} x_{i}^{r}=\sum_{i=1}^{r}\left(x_{i}^{5_{i}^{-1}}\right)^{75}=x .
$$

Thus $\tau$ is identity on $M_{1}^{\prime}[u]$, in particular on $M_{1}^{\prime}$. Since $z$ commutes with $M_{1}^{\prime}, \sigma$ itself is identity on $M_{1}^{\prime}$, hence on $M_{1}$, hence on all of $M: \sigma=1$ and $\tau=I z$. Finally $\tau=1$, because the automorphisms $I(W)$ form a $T$-group on $M_{1}^{\prime}[u]$ over $M_{1}^{\prime}$.

(III) If $x \in N$ is fixed under $I(W)$, it must be in $M^{\prime}$. If it is fixed under $\Delta$ as well, it must be in $K$, since $\Delta$ is a $T$-group on $M$ over $Z$, hence on $M^{\prime}$ over $K$.

We have already observed that the elements $\left\{y_{\sigma} z \mid \sigma \in \Delta, z \in W\right\}$ are proper vectors of $\bar{d}$ :

$$
\bar{d}\left(y_{\sigma} z\right)=y_{\sigma} z(\delta(\sigma)+\lambda) .
$$

Finally, we recall that the degree of $f(x)$ equals $(Z: K)$. Hence $(N: K)=\left(M^{\prime}[u]: K\right)=\left(M^{\prime}: K\right)(Z: K)$. This is precisely the dimension $(M: Z)(Z: K)$ of a maximal commutative subring of the differential extension $B$. Theorem 2 is now established.

REMARK. It is easy to describe the algebra $B$ of Theorem 2 without referring to the structure of a differential extension. Given 
$A=(M, \Delta, \alpha)$ and having chosen $\bar{d}$ and $c$ in such a way that $c \in M^{\prime}$ and $d y_{\sigma}=y_{\sigma} \delta(\sigma)$, as before, we can define a normal ring $(N, \Gamma)$. $N=M^{\prime}[u]$, where $u$ is an indeterminate commuting with elements of $M^{\prime}$ and satisfying the single condition $f(u)=c . \Gamma$ is an extension of the factor group $W / K^{*}$ by $\Delta$ : elements of $\Gamma$ will be written in the form $[\sigma, \zeta]$ with $\sigma \in \Delta, \zeta \in W / K^{*}$. Multiplication is then defined by

$$
[\sigma, \zeta]\left[\sigma^{\prime}, \zeta^{\prime}\right]=\left[\sigma \sigma^{\prime}, \zeta \zeta^{\prime} \alpha\left(\sigma \sigma^{\prime}\right)^{-}\right]
$$

where the bar over an element of $W$ denotes the coset modulo $K^{*}$ to which it belongs. The action of $\Gamma$ on $N$ is given as follows:

$$
\begin{aligned}
& a^{[\sigma, \zeta]}=a^{\sigma} \quad \text { for } a \in M^{\prime}, \\
& u^{[\sigma, \zeta]}=u+\delta(\sigma)+\lambda(\zeta),
\end{aligned}
$$

where $\lambda(\zeta)$ is the one proper value of $d$ common to all representatives of $\zeta$. Finally, $B=(N, \Gamma, \beta)$ with $\beta$ easily computed as

$$
\beta\left([\sigma, \zeta],\left[\sigma^{\prime}, \zeta^{\prime}\right]\right)=\gamma\left(\zeta, \zeta^{\prime}\right) \gamma\left(\zeta \zeta^{\prime}, \alpha\left(\sigma, \sigma^{\prime}\right)^{-}\right) \frac{\alpha\left(\sigma, \sigma^{\prime}\right)}{z_{\alpha\left(\sigma, \sigma^{\prime}\right)^{-}}},
$$

where $\left\{z_{\zeta} \mid \zeta \in W / K^{*}\right\}$ is some fixed system of representatives of $W$ modulo $K^{*}$, and

$$
\gamma\left(\zeta, \zeta^{\prime}\right)=\frac{z_{\zeta} z_{\zeta^{\prime}}}{z_{\zeta \zeta^{\prime}}}
$$

We note especially that the range of $\beta$ is in $K$.

3. Back to the proof of Theorem 1. $E$ is again a regular purely inseparable extension of $K, X$ a maximal regular subgroup of $E^{*}$. We consider the subgroup $W$ of those members of $X$ whose $p$ th power lies in $K^{*}$ and set $Z=K(W)$.

LEMMA. In $E$ over $Z$, the group $X Z^{*}$ is a maximal regular subgroup of $E^{*}$.

Proof. $X$ generates $E$ additively, as before; hence regularity of $X Z^{*}$ over $Z$ is all that must be proved.

Let $\left\{x_{1}, \cdots, x_{s}\right\}$ and $\left\{w_{1}, \cdots, w_{t}\right\}$ be systems of representatives of $X$ modulo $W$ and of $W$ modulo $K^{*}$, respectively. The $K$-space spanned by the latter is clearly a ring and must coincide with $Z$. Since $\left\{x_{i} w_{j} \mid i=1, \cdots, s ; j=1 \cdots t\right\}$ is a system of representatives of $X$ modulo $K^{*}$, it is linearly independent over $K$. Hence $\left\{x_{1}, \cdots, x_{s}\right\}$ is linearly independent over $Z$. This completes the proof because $\left\{x_{1}, \cdots, x_{s}\right\}$ is also a system of representatives of $X Z^{*}$ modulo $Z^{*}$.

We are given a $p$-algebra $A$ over $K$ containing the field $E$ as a 
maximal commutative subring. It is required to show that $A$ also contains a direct sum of fields $N$ with a $T$-group $\Gamma$ of automorphisms which is isomorphic to $G$ and induced by the inner automorphisms of $A$ belonging to a system of representatives of $X$ modulo $K^{*}$. (Here $X$ is an arbitrary preselected maximal regular subgroup of $E^{*}$.) The proof is by induction on the dimension of $E$ over $K$, the assertion being trivial if the latter is 1.

Let $A^{\prime}$ be the centralizer of $Z$ in $A . A^{\prime}$ is central simple over $Z$, contains $E$ as a maximal commutative subring, and has a smaller dimension than $A$. We choose the maximal regular subgroup $X Z^{*}$ of $E^{*}$ for the application of the induction hypothesis. $A^{\prime}$ has the structure of a crossed product $(M, \Delta, \alpha)$, where $(M, \Delta)$ is a normal ring with $\Delta \simeq X Z^{*} / Z^{*} . \Delta$ is induced by the inner automorphisms of $A^{\prime}$ belonging to a system of representatives of $X Z^{*}$ modulo $Z^{*}$ which could certainly be chosen to coincide with the system $\left\{x_{1} \cdots x_{s}\right\}$ occurring in the proof of the lemma. This set was previously denoted by $\left\{y_{\sigma} \mid \sigma \in \Delta\right\}$, and $\alpha(\sigma, \tau)$ defined as $y_{\sigma \tau}^{-1} y_{\sigma} y_{\tau}$. Let the elements of $\Delta$ be numbered $\sigma_{1}, \cdots, \sigma_{s}$ in such a way that $y_{\sigma_{i}}=x_{i}$. We note that $\alpha\left(\sigma_{i}, \sigma_{k}\right) \in W$. W being a maximal regular subgroup of $Z^{*}$, and $A$ (a central simple algebra containing $A^{\prime}$ as the centralizer of $Z$ ) being a differential extension of $A^{\prime}$, we apply Theorem 2 to find a normal ring $(N, \Gamma)$ from which $A$ is produced as a crossed product. The elements of $A^{\prime}$ whose corresponding inner automorphisms induce $\Gamma$ are $\left\{y_{\sigma} z \mid \sigma \in \Delta, z \in W\right\}$ according to the proof of Theorem 2 .

Since $K$ is the center of $A$, the elements $z$ might as well be restricted to the set $\left\{w_{1}, \cdots, w_{t}\right\}$ of representatives of $W$ modulo $K^{*}$. Thus $\Gamma=I(S)$, where $S=\left\{x_{i} w_{j}\right\}$ is a system of representatives of $X$ modulo $K^{*}$. Finally, the map $x K^{*} \rightarrow I x$ is a homomorphism from $X / K^{*}$ onto $\Gamma$; that it is an isomorphism, hence $G \simeq \Gamma$, is most easily seen by noting that $(A: K)=(G: 1)^{2}$.

Theorem 1 is a generalization of one of the central results of [4] (Satz 32), which treats the case where $E$ is a simple extension and $G$ therefore cyclic. Its second formulation, Theorem $1^{\prime}$, is intended to give more equal weight to $E$ and $N$, the former appearing only implicitly in the formula of Theorem 1. It is reminiscent of the simplest $p$-algebras $(\alpha, \beta]$ studied by H. L. Schmid, Witt, and others, which were generated over $K$ by two symbols $u$, $v$ with the relations:

$$
u^{p}=\alpha \in K, \quad v^{p}-v=\beta \in K, \quad u^{-1} v u=v-1 .
$$

We would set $K(u)=E, K(v)=N$, and define $\phi$ by $\phi(\sigma)=u K^{*}, \sigma$ being the automorphism $v \rightarrow v-1$ of $N$. Then $(\alpha, \beta]=E \otimes_{\phi} N$. Whereas criteria for isomorphism and rules about Kronecker products are 
known for the algebras $(\alpha, \beta]$, they remain an open question in the general case.

\section{REFERENCES}

1. K. Hoechsmann, Simple algebras and derivations, Trans. Amer. Math. Soc. (to appear).

2. K. Hoechsmann and H. Zassenhaus, On purely inseparable extensions, Illinois J. Math. (to appear).

3. O. Teichmüller, Verschränkte Produkte mit Normalringen, Deutsche Math. 1 (1936), 92-102.

4. - p-Algebren, Deutsche Math. 1 (1936), 362-388.

5. P. Wolf, Algebraische Theorie der galoisschen Algebren, Mathematische Forschungsberichte, III. VEB Deutscher Verlag der Wissenschaften, Berlin, 1956.

UNIVERSITY OF NOTRE DAME AND

UNIVERSITÄT HAMBURG 